\title{
Penetration of spherical and rod-like gold nanoparticles into intact and barrier-disrupted human skin
}

\author{
Christina Graf ${ }^{\mathrm{a} *}$, Daniel Nordmeyer ${ }^{\mathrm{b}}$, Sebastian Ahlberg ${ }^{\mathrm{b}}$, Jörg Raabe ${ }^{\mathrm{c}}$, Annika Vogt ${ }^{\mathrm{b}}$, Jürgen \\ Lademann $^{\mathrm{b}}$, Fiorenza Rancan ${ }^{\mathrm{b} *}$, Eckart Rühl $^{\mathrm{a}}{ }^{\mathrm{a}}$ \\ ${ }^{a}$ Institut für Chemie und Biochemie - Physikalische und Theoretische Chemie, Freie Universität \\ Berlin, Takustr. 3, 14195 Berlin, Germany, ${ }^{b}$ Department of Dermatology and Allergy, Charité- \\ Universitätsmedizin Berlin, Charitéplatz 1,10117 Berlin, Germany, ${ }^{\mathrm{C} S w i s s ~ L i g h t ~ S o u r c e, ~ P a u l ~}$ \\ Scherrer Institut (PSI), 5232 Villigen, Switzerland
}

\begin{abstract}
The penetration of spherical and rod-like gold nanoparticles into human skin is reported. Several skin preparation techniques are applied, including cryo techniques, such as plunge freezing and freeze drying, and the use of wet cells. Their advantages and drawbacks for observing nanoparticle uptake are discussed. Independent of the particle shape no uptake into intact skin is observed by a combination of imaging approaches, including scanning electron microscopy (SEM), energy dispersive X-ray spectroscopy (EDX), and scanning X-ray microscopy (STXM). These results are discussed along with suitable skin preparation approaches. Experiments on barrier-disrupted skin, i.e. mechanical lesions made by pricking, indicate, however, that gold particles can be identified deep in the dermis, as follows from STXM studies on wet skin samples.
\end{abstract}

Keywords: Scanning Transmission X-Ray Microscopy (STXM), Cryo-Scanning Electron Microscopy (Cryo-SEM), gold nanoparticles, gold nanorods, skin penetration, barrier-disrupted skin

\section{INTRODUCTION}

The use of gold nanoparticles for biomedicine applications has been reviewed recently [1]. Of specific interest are their size- and shape-dependent optical properties, which make these particles interesting for numerous applications beyond life sciences, i.e. photonics and nanoplasmonics including technological applications. In the field of life science it is important that small gold nanoparticles can be probed in biological matter, as has been reported for electron microscopy approaches [2]. Complementary to electron microscopy is X-ray microscopy, which combines high spatial resolution with chemical selectivity, which was recently reviewed by Lawrence et al. [3]. The X-ray contrast of gold has been successfully used for probing the uptake of silica nanoparticles into human skin [4]. In this study, a gold core or gold shells provided the chemical contrast that is required to probe single silica nanoparticles in human skin.

There is general agreement that the uppermost horny layer, the stratum corneum (SC), provides sufficient protection so that nanoparticles can neither be taken up into deeper skin layers nor into the organisms [5-6]. Previous work also highlighted the question, if there are changes in uptake behavior of functionalized silica nanoparticles into skin, if the SC is weakened by tape stripping or by induced allergic dermatitis models [7-8]. Furthermore, the role of hair follicles has been discussed regarding a possible uptake route of nanoparticles and for drug delivery applications [9].

In this study we report on recent results on the uptake of isotropic, i.e. spherical, and anisotropic, i.e. rod-shaped, gold nanoparticles into intact and mechanically damaged human skin samples. The motivation of this work is to complement recent work on functionalized silica nanoparticles, which have indicated that intact as well as even tape-stripped skin and induced allergic dermatitis models do not show any evidence for nanoparticle uptake [7-8]. 


\section{METHODOLOGY}

\subsection{Synthesis and characterization of gold nanoparticles}

Cetyl trimethylammonium bromide (CTAB)-coated rod-like gold nanoparticles were prepared by a reported procedure [10]. Subsequently, negatively charged polystyrene sulfonate (PSS) chains were wrapped around the particles as described in detail by Pastoriza-Santos et al. [11]. Subsequently, the gold nanorods were purified by repeated centrifugation and redispersion in ultrapure water to remove excess CTAB. Mostly spherical, citrate-coated gold nanoparticles in aqueous dispersion were obtained from BBI Solutions (Cardiff, UK) and were used as received. Both samples were characterized by electron microcopy using a Hitachi SU8030 SEM in the STEM mode operating at an acceleration voltage of $30 \mathrm{kV}$ and after adjusting the gold concentration to $3.0 \pm 0.1 \mathrm{~g} / \mathrm{L}$ by dynamic light scattering and zeta potential measurements (Delsa Nano C) [12].

\subsection{Preparation of skin samples}

Human skin explants from the abdomen were obtained from patients undergoing plastic surgery, after their approved consent and approval by the ethic committee of the Charite Universitätsmedizin Berlin (Germany). The human skin explants were pricked with a lancet, whereas control samples remained untreated. Aqueous dispersions of the gold nanorods or gold nanospheres $(3.0 \pm 0.1 \mathrm{~g} / \mathrm{L})$ were dropped on the skin samples, homogenously distributed, and incubated for $18 \mathrm{~h}$. Subsequently, the top layers of the stratum corneum were removed by tape-stripping with five tapes. Skin pieces of $3 \mathrm{~mm} \times 3 \mathrm{~mm}$ were cut for the investigations.

The skin blocks were plunge frozen in liquid ethane and subsequently placed in a tissue freezing medium (Leica Microsystems, Germany). These samples were then cut at $-20^{\circ} \mathrm{C}$ to slices of 3-5 $\mu \mathrm{m}$ thickness using a microtome (2800 Frigocut-N, Reichert-Jung, Heidelberg, Germany). Two different procedures were applied for preparing samples for microscopy studies: (i) The frozen samples were then transferred on double folding mesh Cu-TEM grids (Plano) for investigations by cryo-SEM at $-130^{\circ} \mathrm{C}$. The samples were freeze-dried in the SEM by step-wise raising the temperature from $-130^{\circ} \mathrm{C}$ to room temperature at $\mathrm{p} \sim 10^{-3} \mathrm{~Pa}$, and finally they were investigated as dried samples at the same pressure by scanning transmission X-ray microscopy (STXM) [4]; (ii) The frozen samples were transferred to a silicon nitride membrane windows (Silson, UK), warmed up to room temperature, fixed with $2 \%$ paraformaldehyde (PFA), and covered with a second silicon nitride grid. Then, the samples were sealed by nail polish and investigated in a helium atmosphere $\left(\mathrm{p}=5 \cdot 10^{4} \mathrm{~Pa}\right)$ by STXM.

\subsection{Cryo-Scanning Electron Microscopy (Cryo-SEM) experiments}

Cryo-SEM was carried out using a Hitachi SU 8030 SEM, equipped with a Gatan Alto 2500 cryo-transfer system and an Oxford X-Max $80 \mathrm{~mm}^{2}$ EDX detector. Frozen skin sections on TEM grids were mounted onto the cryo-transfer holder in a liquid nitrogen bath, and were then transferred into the $-130^{\circ} \mathrm{C}$ cooled preparation chamber of the Gatan Alto 2500 under vacuum conditions $\left(\mathrm{p} \approx 10^{-3} \mathrm{~Pa}\right.$ ). Superficial ice was removed by sublimation $(\mathrm{t} \approx 3 \mathrm{~min})$ at a temperature of $-100^{\circ} \mathrm{C}$ at $\sim 10^{-3} \mathrm{~Pa}$. Subsequently, the sample was cooled to $\sim-130^{\circ} \mathrm{C}$, and examined at an accelerating voltage of $10 \mathrm{kV}$ at -130 ${ }^{\circ} \mathrm{C}$. EDX measurements were carried out under the same conditions. Finally, the samples were transferred back into the preparation chamber and the temperature was step-wise increased to room temperature. The samples were studied again by SEM in order to identify possible damage during this drying procedure.

\subsection{Scanning Transmission X-Ray Microscopy (STXM) experiments}

The measurements were performed on the PolLux scanning transmission soft X-ray microscopy (STXM) microscope at the Swiss Light Source (see ref. [4] for details). The samples were mounted vertically on a high-precision piezoelectric stage and scanned in order to take two-dimensional images of the samples. The instrument is operated under vacuum $\left(10^{-1} \mathrm{~Pa}\right)$ or in a helium atmosphere $\left(\mathrm{p}=5 \cdot 10^{4} \mathrm{~Pa}\right)$ in order to minimize the X-ray absorption by air in the soft X-ray regime, as well as to avoid sample contamination. All images were recorded below the $\mathrm{O} 1 \mathrm{~s}$ regime at $510 \mathrm{eV}$. This provides strong X-ray absorption contrast by the gold nanoparticles. 


\section{RESULTS DISCUSSION}

\subsection{Synthesis and characterization of gold nanoparticles}

Mainly rod-like nanoparticles with a high aspect ratio were obtained as described in Section 2.1 (see Figure 1 (A) and TEM data in Table 1). The sample contains also a small amount $(<5 \%)$ of spherical and prismatic-triangular particles. The subsequent surface coating with PSS does not influence the size, shape, and colloidal stability of the particles but turns their zeta potential from positive into highly negative values (cf. Table 1), which is known to enhance the cell uptake [12]. Note that in this coating procedure CTAB was not removed from the surface as no ligand exchange, rather than an electrostatic adsorption process took place. However, excess free CTAB was significantly removed by copious purification of the particles, so that the concentration of free CTAB is expected to be negligible. The citrate-coated gold nanoparticles from the commercial supplier BBI Solutions (Cardiff, UK) were almost spherical in shape. However, a significant fraction of $\sim 8 \%$ of prismatic-triangular and slightly elongated nanoparticles was also present (see TEM image in Figure 1 (B)). These particles are nearly monodisperse and have as a high colloidal stability (see Table 1).

\subsection{Skin sample preparation and characterization}

Human skin explants were either pricked or left untreated before they were incubated for $18 \mathrm{~h}$ with aqueous dispersions of the nanoparticles. This allowed us to investigate the uptake of differently shaped, negatively charged gold nanoparticles in intact and barrier-disrupted human skin samples. A superficial tape strip was finally applied in order to remove all particles which have not penetrated the top layers of the stratum corneum.

The aim of the present work was to determine the penetration efficiency of differently shaped nanoparticles in human skin. This required on the one hand high spatial resolution, which is ideally combined with chemical contrast allowing to identify chemical changes at and close to the nanoparticles as well as possible changes of tissue and cells. On the other hand, the tissue should be as less influenced as possible by the preparation process. We have applied for this work the label-free approaches SEM and STXM, which require no staining of the sample. SEM has the advantage of a higher spatial resolution than STXM (1 nm for SEM at room temperature and about $5 \mathrm{~nm}$ for cryo-SEM, respectively, as compared to $\sim 15 \mathrm{~nm}$ for STXM). However, chemical contrast is relatively low due to energy resolution of EDX, which is used along with SEM. This allows us to distinguish different chemical elements but there is no chemical contrast for compounds consisting of the same elements. Moreover, SEM requires high vacuum and, hence, tissue samples are usually investigated as cryogenic samples at low temperatures $\left(-130^{\circ} \mathrm{C}\right)$.

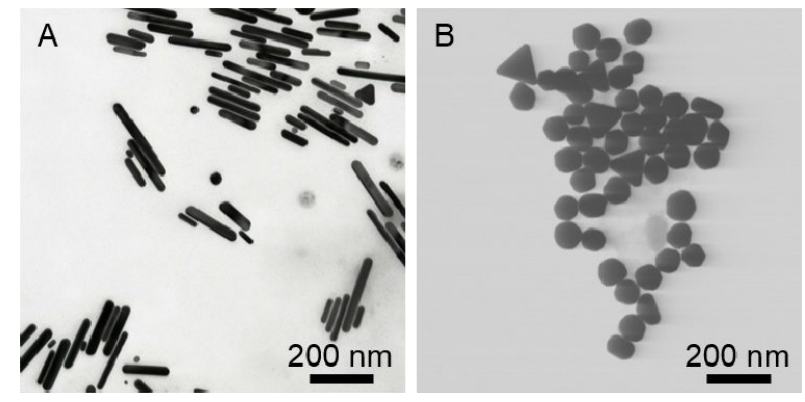

Figure 1. Transmission electron micrgraphs of (A) polystyrene sulfonate (PSS)-coated rod-like gold nanoparticles and (B) spherical, citrate-coated gold nanoparticles.

Table 1. Characterization of the gold nanoparticles used in this work.

\begin{tabular}{|l|c|c|c|c|}
\hline \multicolumn{1}{|c|}{ Sample } & TEM size & $\begin{array}{c}\text { Hydrodynamic } \\
\text { diameter } \\
\text { [nm] }\end{array}$ & $\begin{array}{c}\text { Polydispersity } \\
\text { index } \\
\text { [nm] }\end{array}$ & Zeta Potential \\
[meV]
\end{tabular}


Alternatively, dried or resin-embedded samples can be studied by SEM. In contrast, STXM has a lower spatial resolution $(\sim 15 \mathrm{~nm})$ than SEM but X-ray spectromicroscopy provides a significantly higher chemical contrast than EDX. This allows for identification of different chemical compounds or chemical transformations, if the photon energy is tuned across near-edge spectra [3]. In addition, STXM allows for the investigation of wet samples since measurements in a helium atmosphere at relatively high pressure are feasible to perform. By contrast, wet samples cannot be easily investigated by SEM, so that there is a complementarity of both approaches. Note that cryo-STXM is not routinely available at all synchrotron radiation facilities (cf. [13]), so that the present work was performed at ambient temperature. Moreover, STXM requires thin samples, which have typically a thickness of a few $\mu \mathrm{m}$ or less. Such samples can be only obtained from slicing frozen skin or fixed skin embedded in resin. Embedding skin in resin may cause dislocation of nanoparticles during the preparation process, whereas freezing and thawing the sample may damage cells and tissue. Consequently, two different strategies were applied in this work to investigate the uptake of the nanoparticles in the skin by high resolution microscopy techniques, where the advantages and disadvantages of each approach are briefly elucidated: (i) The skin samples were plunge-frozen at $-130^{\circ} \mathrm{C}$, which guarantees minimal damage of the cells. The samples were subsequently carefully warmed up to $-20^{\circ} \mathrm{C}$ for slicing. Then they were again cooled down to $-130^{\circ} \mathrm{C}$ for the investigation by cryo-SEM. Subsequently, the samples were freeze-dried and studied again by SEM so that identical samples were also investigated by STXM. In this way, exactly the same skin areas can be investigated by SEM and STXM. Notably, the SEM samples were not sputtered with platinum, since this would impede freeze-drying as well as any investigation by STXM. Furthermore, Pt and Au have nearly identical EDX spectra and cannot be distinguish from each other by EDX; (ii) The skin samples were also plunge-frozen at $-130^{\circ} \mathrm{C}$ and sliced at $-20^{\circ} \mathrm{C}$ but then they were warmed up, fixed, and investigated as wet samples in a sealed cell at room temperature in a helium atmosphere by STXM. In this case, SEM investigations on the same samples studied by STXM could not be carried out.

\subsection{Cryo-Scanning Electron Microscopy (Cryo-SEM) and Scanning Transmission X-Ray Microscopy (STXM) experiments on frozen and freeze-dried skin samples}

Figure 2 (A) shows a typical image of intact frozen skin at $-130^{\circ} \mathrm{C}$ that was exposed to gold nanorods. The stratum corneum (SC) as well as the cells in the epidermis (E) can be easily identified. Since the gold nanorods are rather thin (20 nm, cf. Table 1) and mostly not oriented with their longitudinal axis parallel to the image plane, it is hard to identify single rods by this approach. Therefore, EDX measurements were carried out. In Figure 2 (A) no gold could be detected by EDX, which is in agreement with earlier results indicating that nanoparticles in this size range $(50 \mathrm{~nm}$ diameter and larger) cannot penetrate through the intact stratum corneum [4]. Figure 2 (B) shows another area of the same skin sample where a deep prick site (P), i.e. mechanical damage of the skin, is present. An EDX analysis of this image shows that a significant amount of gold, i.e. gold nanorods, is present in this area (see Figure 3 (C)). Note that $\mathrm{C}$ and $\mathrm{O}$ are detected by EDX, as well (see Figure 2 (B)), which comes mostly from the skin sample. The $\mathrm{Cu}$ and $\mathrm{Zn}$ signals originate from the $\mathrm{Cu}$ TEM grid and the sample holder. Both signals are even present if no skin sample is present in the SEM chamber.
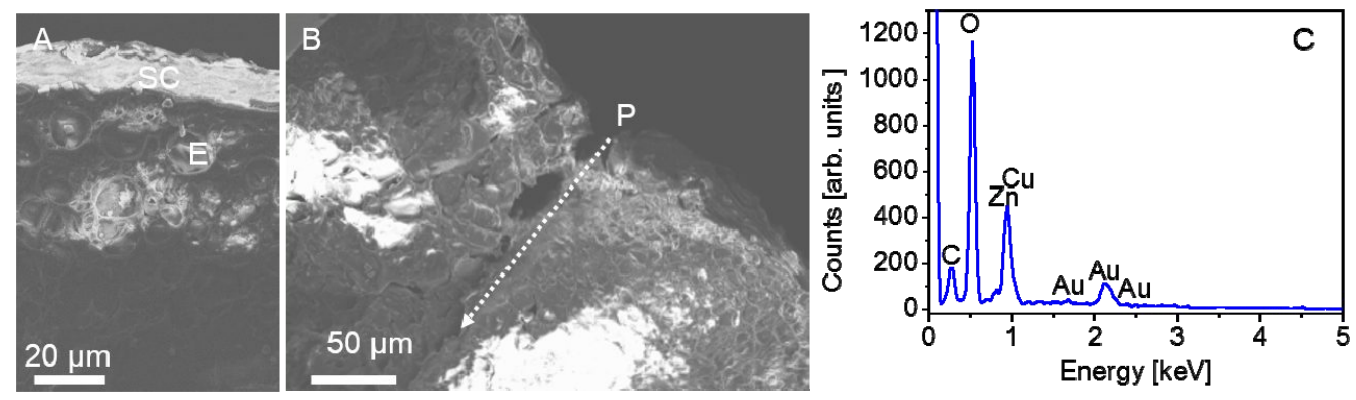

Figure 2. Cryo-SEM images of (A) intact and (B) pricked human skin; (C) EDX spectrum of image (B) indicating the presence of gold nanorods in the pricked skin.

Figure 3 (A) shows a cryo SEM image of pricked skin where a significant amount of gold nanospheres is present as it is proven by the corresponding EDX spectrum of this area (see Figure 3 (B)). This sample was then carefully freeze-dried for the subsequent STXM measurements. However, the freeze-drying procedure significantly damaged the skin (see TEM image in Figure $3(\mathrm{C})$ ). The skin shows numerous holes because sublimation of ice leads to damage of the cell 
membranes. Before the freeze-drying it was impossible to investigate this area by TEM because it was not sufficiently transparent for this technique and, hence, the skin sample appeared to be opaque for the electron beam. This sample was then investigated by STXM (see Figures 2 (D)-(F)) and indeed gold nanospheres could be identified (see small spheres in Figure $2(\mathrm{~F}))$ at the skin surface in the stratum corneum. However, in other parts of this sample no gold nanoparticles where found by STXM. Subsequent EDX studies provided evidence for the fact that during the freeze-drying process gold nanoparticles got lost. Consequently, the freeze-drying approach is not well-suited for preparation of skin samples for STXM studies, whereas the combined SEM/EDX studies appeared to be a useful approach for locating areas containing gold nanoparticles in skin.
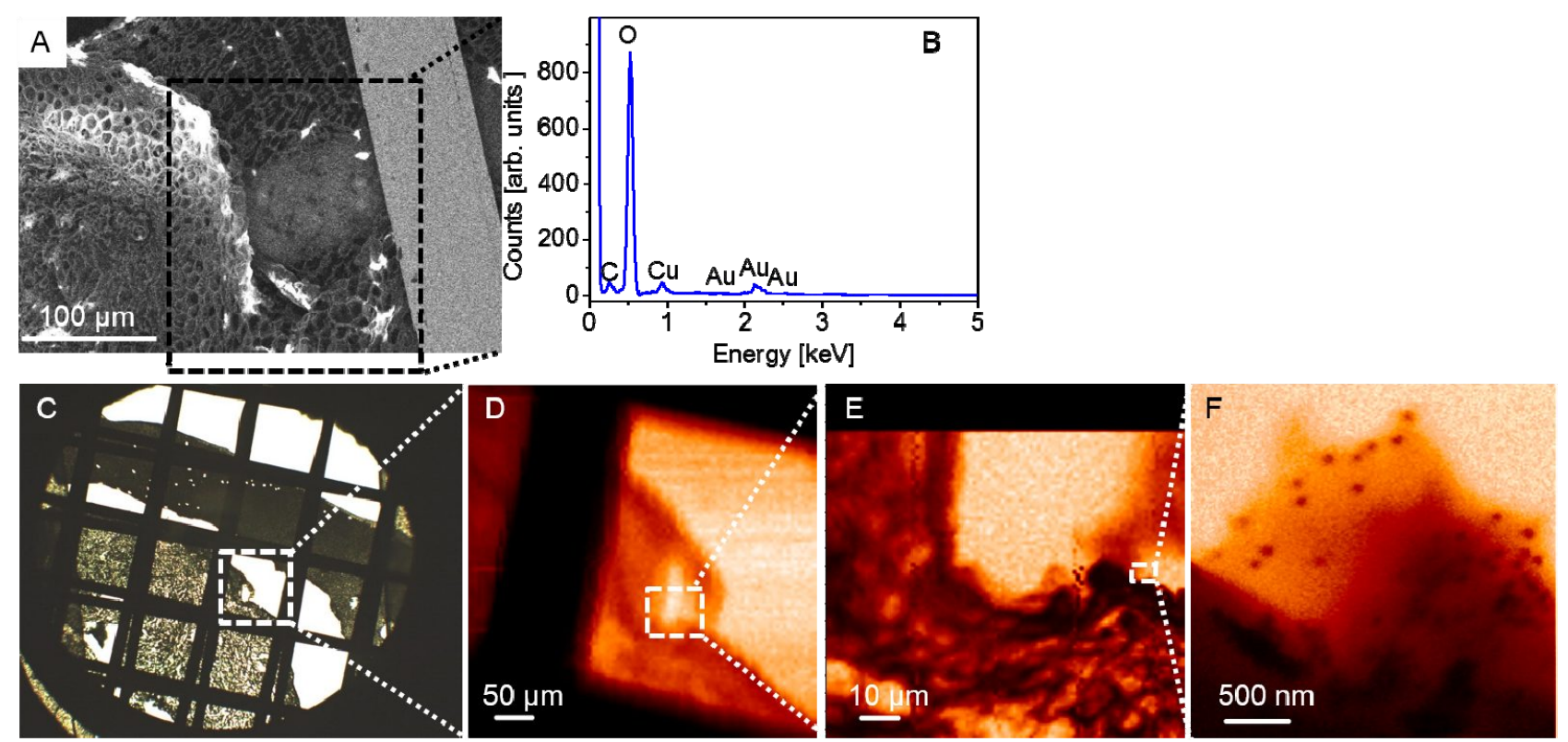

Figure 3. (A) Cryo-SEM image and corresponding (B) EDX spectrum of pricked human skin incubated with gold nanospheres before freeze drying; (C) STEM image of the same sample after freeze-drying and (D) - (F) STXM images of the same freeze-dried sample. STXM allows the identification of single nanoparticles located in the stratum corneum (small dots in Figure (F)).

\subsection{Scanning Transmission X-Ray Microscopy (STXM) experiments on wet skin samples}

Native skin samples were investigated in a wet cell containing a helium atmosphere, since the freeze-drying approach was not advantageous for STXM studies, as outlined in the previous Section. The samples were initially characterized by optical microscopy (see Figure 4 (A)) and then regions of interest were imaged by STXM (see Figure 4 (B) and 4 (C)).

In the STXM images gold nanorods could be clearly identified inside the prick site, i.e. the mechanically-induced lesion of skin. This is due to the fact that the stratum corneum is damaged and the other skin layers do not provide a barrier for transport of the nanorods. As a result, the nanoparticles may diffuse without significant hindrance into the skin. 

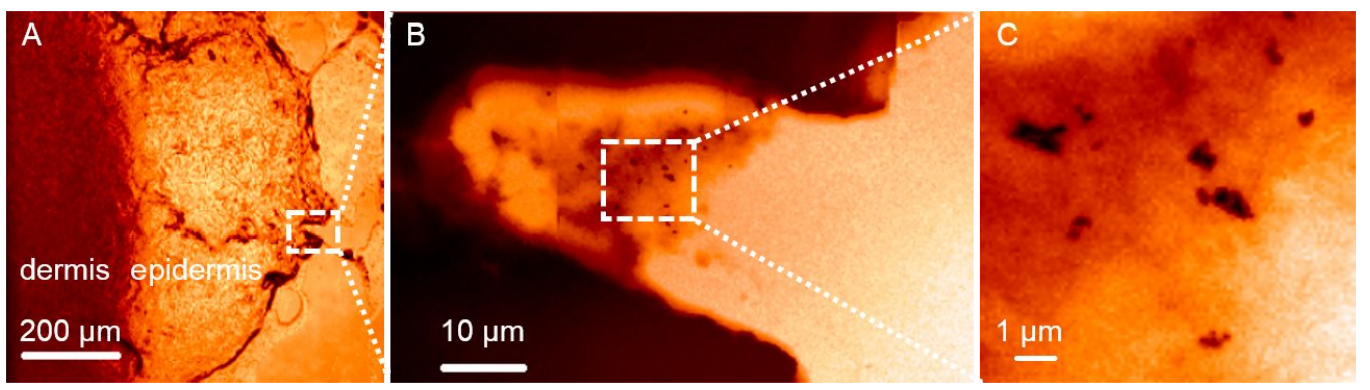

Figure 4. (A) Optical microscopy image and (B) and (C) STXM images at E $=510 \mathrm{eV}$ of pricked human skin incubated for $18 \mathrm{~h}$ with gold nanorods. The sample was investigated in a wet chamber at ambient temperature in a helium atmosphere. STXM allows for the identification of single gold rods in the stratum corneum (see images (C)).

It was also investigated whether the gold nanorods can penetrate deeper into the epidermis or dermis, if they have passed the stratum corneum at the prick site. This situation models the case of mechanical skin injuries, which come into contact with dispersions of inorganic nanoparticles, such as nanoparticle-based sunscreens. Therefore, two areas near the injury which are located at approximately 100 and $50 \mu \mathrm{m}$ away from the prick site (P) (see Figure 5(A), insets 1 and 2) were studied in greater detail. Indeed, gold nanorods could be found in these areas that are located in the dermis (see Figure 5 (B) and $5(\mathrm{C})$ ) for area 1 as well as Figure 5 (D) - (F) for area 2. These results demonstrate that gold nanorods can be identified in regions that are located deep in the dermis, as probed by high resolution STXM.
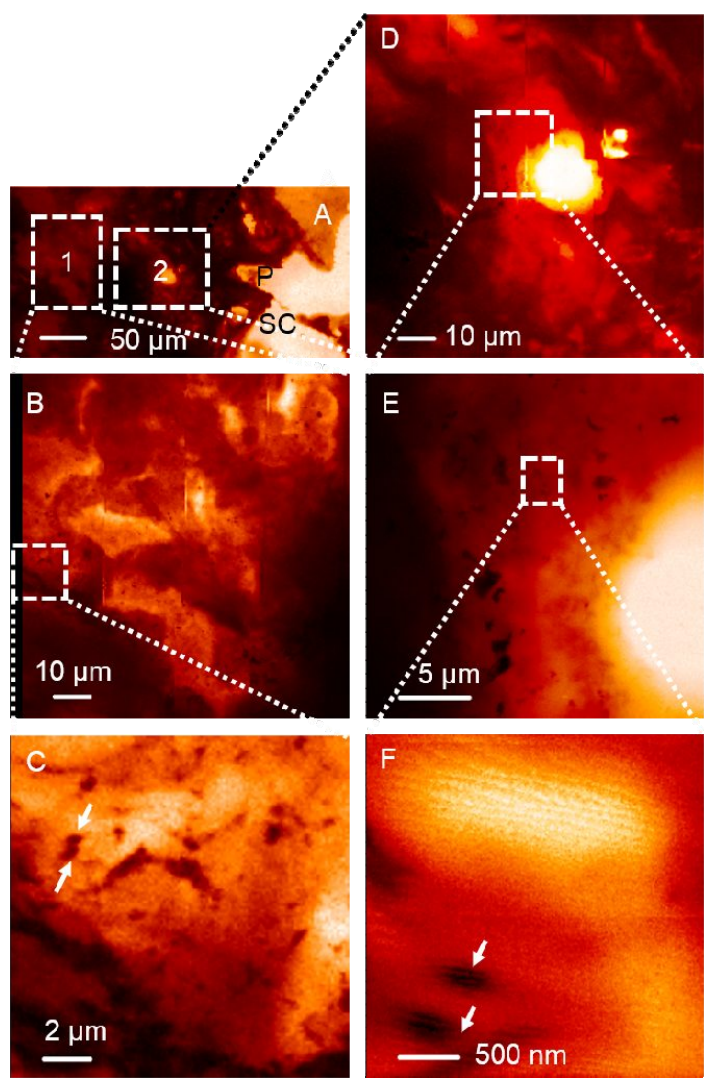

Figure 5. STXM images $(E=510 \mathrm{eV})$ of the same skin sample as shown in Figure 4 where gold nanorods penetrate deep into the dermis below the injury caused by pricking (P) of the skin, i.e. stratum corneum (SC) and parts of the viable epidermis. Image (A) shows an overview, the images (B) and (C) are enlarged views of area 1, the images (D), (E), (F) show enlarged views of area 2. STXM allows for the identification of gold rods in the dermis (see arrows in images (C) and (F)). 


\section{CONCLUSIONS}

The uptake of differently shaped gold nanoparticles is investigated by scanning electron and X-ray microscopy. Evidence is provided for the result that neither the particle size nor the shape are of importance for the efficient transfer through the skin barrier, which is provided by the stratum corneum. It is shown that only massive mechanical damage of the skin barrier by pricking leads to the uptake of nanoparticles into the dermis. We have shown that skin preparation is of crucial importance for investigating the uptake of nanoparticles by scanning electron and X-ray microscopy. In general, advanced cryo sample preparation techniques are advantageous for avoiding the manipulation of biological tissue samples by embedding in resins. It is shown that freeze drying of the skin samples is associated with the problem that nanoparticles may get lost. It is also shown that pricked skin samples studied by X-rays microscopy in wet cells are able to probe the penetration of nanorods in the dermis with high spatial resolution along with chemical contrast provided by tunable X-rays. Finally, it is pointed out that X-ray microscopy appears to be a promising approach for studying not only penetration of inorganic nanoparticles, but also drug delivery processes of substances and drug carriers that can penetrate the intact or damaged skin barrier. This is essentially due to the chemical selectivity of this method.

\section{ACKNOWLEDGMENTS}

Financial support by the German Research Foundation (DFG) is gratefully acknowledged (SPP 1313, NANO-SELECT Network) as well as SFB 1112. We thank Dr. Michael Laue from Robert Koch Institute for plunge-freezing of the skin samples and fruitful discussions.

\section{REFERENCES}

[1] Dreaden, E. C.; Alkilany, A. M.; Huang, X.; Murphy, C. J.; El-Sayed, M. A., "The golden age: gold nanoparticles for biomedicine," Chem. Soc. Rev. 41, 2740-2779 (2012).

[2] Goldstein, A.; Soroka, Y.; Frusic-Zlotkin, M.; Popov, I.; Kohen, R., "High resolution SEM imaging of gold nanoparticles in cells and tissues," J. Microsc. 256, 237-247 (2014).

[3] Lawrence, J. R.; Swerhone, G. D. W.; Dynes, J. J.; Korber, D. R.; Hitchcock, A. P., "Soft X-ray spectromicroscopy for speciation, quantitation and nano-eco-toxicology of nanomaterials," J. Microsc., in print (2015).

[4] Graf, C.; Meinke, M.; Gao, Q.; Hadam, S.; Raabe, J.; Sterry, W.; Blume-Peytavi, U.; Lademann, J.; Rühl, E.; Vogt, A., "Qualitative detection of single submicron and nanoparticles in human skin by scanning transmission x-ray microscopy," J. Biomed. Opt. 14, 021015 (9 pages) (2009).

[5] Darvin, M. E.; Konig, K.; Kellner-Hoefer, M.; Breunig, H. G.; Werncke, W.; Meinke, M. C.; Patzelt, A.; Sterry, W.; Lademann, J., "Safety Assessment by Multiphoton Fluorescence/Second Harmonic Generation/HyperRayleigh Scattering Tomography of ZnO Nanoparticles Used in Cosmetic Products," Skin Pharmacology and Physiology 25, 219-226 (2012).

[6] Rancan, F.; Gao, Q.; Graf, C.; Troppens, S.; Hadam, S.; Hackbarth, S.; Kembuan, C.; Blume-Peytavi, U.; Rühl, E.; Lademann, J.; Vogt, A., "Skin Penetration and Cellular Uptake of Amorphous Silica Nanoparticles with Variable Size, Surface Functionalization, and Colloidal Stability," Acs Nano 6, 6829-6842 (2012).

[7] Ostrowski, A.; Nordmeyer, D.; Boreham, A.; Brodwolf, R.; Mundhenk, L.; Fluhr, J. W.; Lademann, J.; Graf, C.; Rühl, E.; Alexiev, U.; Gruber, A. D., "Skin barrier disruptions in tape stripped and allergic dermatitis models have no effect on dermal penetration and systemic distribution of AHAPS-functionalized silica nanoparticles," Nanomed. Nanotechnol. 10, 1571-1581 (2014).

[8] Ostrowski, A.; Nordmeyer, D.; Mundhenk, L.; Fluhr, J. W.; Lademann, J.; Graf, C.; Rühl, E.; Gruber, A. D., "AHAPS-functionalized silica nanoparticles do not modulate allergic contact dermatitis in mice," Nanoscale Res. Lett. 9, 524 (7 pages) (2014).

[9] Lademann, J.; Patzelt, A.; Richter, H.; Lademann, O.; Baier, G.; Breucker, L.; Landfester, K., "Nanocapsules for drug delivery through the skin barrier by tissue-tolerable plasma," Laser Physics Letters 10, 083001 (2013).

[10] Nikoobakht, B.; El-Sayed, M. A., "Preparation and Growth Mechanism of Gold Nanorods (NRs) Using SeedMediated Growth Method," Chem. Mater. 15, 1957-1962 (2003). 
[11] Pastoriza-Santos, I.; Perez-Juste, J.; Liz-Marzan, L. M., "Silica-Coating and Hydrophobation of CTABStabilized Gold Nanorods," Chem. Mater. 18, 2465-2467 (2006).

[12] Graf, C.; Gao, Q.; Schütz, I.; Njiki Noufele, C.; Ruan, W.; Posselt, U.; Korotianskiy, E.; Dordmeyer, D.; Rancan, F.; Hadam, S.; Vogt, A.; ademann, J.; Haucke, V.; Rühl, E., "Surface Functionalization of Silica Nanoparticles Supports Colloidal Stability in Physiological Media and Facilitates Internalization in Cells," Langmuir 28, 7598-7613 (2012).

[13] Wang, Y.; Jacobsen, C.; Maser, J.; Osanna, A., "Soft X-ray microscopy with a cryo scanning transmission Xray microscope: II. Tomography," J. Microsc. 197, 80-94 (2000). 